\title{
Editorial
}

Raafat S. Hannallah MD FRCPC

\section{Postoperative analgesia in the paediatric patient}

Until recently, there has been a widespread belief that children do not experience pain and therefore seldom need analgesic medications after most surgical procedures. As a result, either no analgesics were used, or the dose or route recommended was inadequate to provide effective pain relief.

In the last 15 yr there has been a revolutionary growth in the understanding and management of paediatric postoperative pain. This has resulted in the availability of an abundance of therapeutic modalities that now exist to prevent and treat pain, as well as a general acceptance that acute postoperative pain management is an integral part of the practice of paediatric anaesthesia.

This issue of the Journal contains an article by Watcha et al. entitled "Perioperative effects of oral ketorolac and acetaminophen in children undergoing bilateral myringotomy." This paper underscores many timely issues in the field of postoperative analgesia in the paediatric population that warrant special attention.

The fact that the authors chose to study children undergoing myringotomies is in itself very important. Anaesthetists have come a long way from the days when analgesics were seldom prescribed for even more painful surgical procedures, e.g., circumcision and herniotomy. As Watcha has demonstrated, some children experience pain or discomfort even after one of the least invasive of surgical procedures.

Paediatric pain assessment and measurement continues to be an area of controversy. Several methods for assessing pain in children have been developed; none, however, is completely satisfactory especially in preverbal children. The Objective Pain Scale used in this study is a numerical rating scale $(0-10)$ that incorporates both physiological and behavioural responses to pain. Other available pain assessment tools include the CHEOPS, which is a behavioural rating scale, as well as a variety of self-report

Departments of Anesthesiology and Pediatrics, Children's National Medical Center and George Washington University, Washington, D.C. 20010, USA. scales that use graphic modifications of the original linear Visual Analogue Scale. Although these scales have been shown to have good interobserver and concurrent validity, their usefulness for therapeutic decision-making has not been standardized.

The recent trend in postoperative pain management has been to get away from standard formulae, and to titrate drugs to effect. Regional blocks or infiltration of the incision with local anaesthetic solution should be used whenever possible. In the inpatient, Patient-Controlled Analgesia (PCA) has been used in children as young as six years of age. For ambulatory patients, intravenous titration of short-acting narcotic analgesics has replaced standard or routine dosing regimens in most paediatric centres.

The recent introduction of a parenteral preparation of the nonsteroidal anti-inflammatory drug (NSAID) ketorolac tromethamine is another small but important advance in the effort to achieve pain relief without narcosis in children. Several areas of concern must be addressed before ketorolac finds its ultimate place in postoperative paediatric pain management. First, more prospective randomized double-blind studies are needed to establish the safety and efficacy of this drug in children. The manufacturer has an obligation to sponsor such studies and to seek a formal paediatric indication for its use. Not to do so, puts the onus on the individual physician to prove that the use of the drug in a particular patient was justified. The same is true for obtaining quick approval for its intravenous use. Second, in view of the present high cost and scarcity of ketorolac, further studies must carefully evaluate the analgesic properties of this drug against equipotent doses of other available analgesics. Watcha et al. found that oral ketorolac $\left(1 \mathrm{mg} \cdot \mathrm{kg}^{-1}\right)$ was a more effective analgesic than acetaminophen $\left(10 \mathrm{mg} \cdot \mathrm{kg}^{-1}\right)$ or placebo in children undergoing myringotomies. Would either a higher dose of acetaminophen (e.g., 20 or 30 $\mathrm{mg} \cdot \mathrm{kg}^{-1}$ ) or an equipotent dose of a much less expensive NSAID such as ibuprofen have been equally effective? Ketorolac is not without side effects. The apparent increase in postoperative vomiting is a concern. The clinical significance of the drug's effect on platelet function must 
be evaluated objectively in a large number of patients before its routine use in children who are prone to postoperative haemorrhage, such as following tonsillectomy, can be recommended. The eventual place of this, and other NSAIDS that will follow, is a question that will be answered only by large-scale clinical trials that compare objectively their safety, efficacy and cost-effectiveness with existing, well-established methods of providing postoperative analgesia.

\section{La douleur postopératoire chez les enfants}

Jusqu'à récemment on préférait croire que les enfants ne percevaient pas la douleur et qu'ils n'avaient que rarement besoin d'analgésique après une intervention chirurgicale. On ne leur en donnait donc pas, ou encore si peu, qu'une posologie efficace demeurait horst d'atteinte. Toutefois depuis quinze ans, on comprend et traite mieux la douleur consécutive à la chirurgie pédiatrique. Plusieurs stratégies d'intervention permettent maintenant la prévention et le soulagement de la douleur postopératoire et elles font désormais partie de l'arsenal de l'anesthésiste pédiatrique.

Vous lirez plus loin dans ce numérodu Journal, un article de Watcha et al. portant sur les effets péri-opératoires du kétorolac et de l'acétaminophène lors de myringotomies bilatérales chez les enfants, où ils soulig-nent plusieurs aspects importants de l'analgésie postopératoire pédiatrique. Il est d'ailleurs significatif que les auteurs ailent choisi comme cible la douleur provoquée par une intervention chirurgicale aussi bénigne, alors qu'il n'y a pas si longtemps, les anesthésistes ne portaient que peu d'attention à des opérations aussi algogènes que la circoncision et l'herniotomie. Du reste dans cette étude, Watcha et al. rapportent que même une intervention des plus banales peut déclencher de la douleur chez l'enfant.

La mesure et l'évaluation de la douleur en pédiatrie porte encore à controverse. Même si on a conçu plusieurs méthodes pour évaluer cette douleur, aucune ne fonctionne réellement bien, surtout sur des enfants qui n'ont pas atteint l'âge de la parole. L'échelle objective de la douleur employée pour cette étude est graduée de une à dix et tient compte des réactions physiologiques et comportementales qui accompagnent la douleur. Il existe d'autres échelles à dominante comportementale, telle le CHEOPS, et encore d'autres de type auto-évaluation, qui sont des variantes graphiques de l'échelle visuelle analogique originale. Même si la reproductibilité de ces échelles est adéquate, on n'a pas encore éprouvé leur valeur initiale en vue d'une décision thérapeutique.

En analgésie postopératoire, on a tendance, dans la pluppart des centres pédiatriques, à oublier les posologies standardisées pour plutôt titrer les doses d'analgésiques jusqu'à l'effet escompté; on emploie ainsi avec succès des petites doses de morphiniques à courte durée d'action par voie intraveineuse chez les patients ambulatoires. Autant que possible, on se sert des anesthésiques locaux pour réaliser des blocs régionaux ou infiltrer les lèvres d'une plaie. Chez les patients hospitalisés, l'analgésie autoadministrée (PCA) s'avère utile même chez des enfants aussi jeunes que six ans.

L'avènement récent d'une préparation parentérale d'anti-inflammatoire non-stéroïdien (AINS), le trométhamine de kétorolac signifie un pas de plus vers une analgésie pédiatrique de qualité sans sédation. Toutefois, plusieurs questions doivent être élucidées avant de laisser se généraliser l'usage du kétorolac. On doit d'abord multiplier les essais cliniques à double-insu afin de confirmer l'efficacité et la sécurité du kétorolac chez les enfants. Le manufacturier doit parrainer ces études et obtenir une reconnaissance officielle des indications pédiatriques de ce médicament. Il doit aussi chercher activement à en faire approuver l'administration intraveineuse. Autrement, les cliniciens seuls devront porter la responsabilité de son utilisation au jour le jour. Par ailleurs, les coûts du kétorolac et sa rareté requièrent qu'on compare ses propriétés analgésiques avec celles des autres médicaments disponibles. Watcha et al. ont trouvé que le kétorolac oral à raison de $1 \mathrm{mg} \cdot \mathrm{kg}^{-1}$ était un analgésique plus efficace que l'acétaminophène à $10 \mathrm{mg} \cdot \mathrm{kg}^{-1}$ et un placébo pour la myringotomie chez l'enfant. Une plus forte dose d'acétaminophène $\left(20,30 \mathrm{mg} \cdot \mathrm{kg}^{-1}\right)$ aurait-t-elle suffi? Qu'en est-il d'une dose équivalente d'un AINS moins coûteux comme l'ibuprofène? Enfin, il nous faut aussi bien évaluer les effets secondaires du kétorolac: l'apparente augmentation des vomissements postopératoires reste à clarifier de même que l'effet possible du médicament sur la fonction plaquettaire avant qu'on puisse en recommander l'usage pendant l'amygdalectomie où un risque d'hémorragie postopératoire existe. La place de ce nouvel analgésique, et celle des autres AINS qui suivront sans doute, ne sera déterminée qu'à la suite d'essais cliniques de grande envergure qui en compareront objectivement la sécurité et l'efficacité avec celles des autres méthodes d'analgésie postopératoire déjà passées dans nos moeurs. 